\title{
Positron annihilation study of aluminum, titanium, and iron alloys surface after shot peening
}

\author{
R. Zaleski ${ }^{1}$ (1) $\cdot$ K. Zaleski ${ }^{2} \cdot$ M. Gorgol ${ }^{1} \cdot$ M. Wiertel $^{1}$
}

Received: 25 February 2015/Accepted: 3 May 2015/Published online: 17 May 2015

(c) The Author(s) 2015. This article is published with open access at Springerlink.com

\begin{abstract}
Shot peening influence on alloys based on iron, aluminum, and titanium was studied using positron annihilation lifetime spectroscopy (PALS) and residual stress measurements. The PALS spectra were analyzed assuming two lifetime components. While the residual stresses change in a similar way in all the samples, the PALS results show an opposite tendency of a component relative intensities change with the time of shot peening for the $\mathrm{Ti}$ alloy as compared to steel or the $\mathrm{Al}$ alloy. A comparison between the depth profiles of positron implantation and the residual stress distribution reveals that the positron range covers a whole depth where residual stress is observed only in the Ti alloy. Based on this observation, the evolution of the defect concentration is presumed, consisting in migration of large defects away from the surface, while only smaller ones remain close to the surface. Furthermore, the positron lifetime distribution in the Al alloy was determined using the MELT program. The results showed that the initial single, wide distribution of lifetime splits into two narrower ones with increasing shot peening time.
\end{abstract}

\section{Introduction}

The safety of large-scale structures (e.g., nuclear reactors or aircrafts) attracts considerable attention. In order to improve the safety of such systems, a verification of material

\section{R. Zaleski}

radek@zaleski.umcs.pl

1 Institute of Physics, Maria Curie-Sklodowska University, pl. Marii Curie-Sklodowskiej 1, 20-031 Lublin, Poland

2 Faculty of Mechanical Engineering, Lublin University of Technology, ul. Nadbystrzycka 36, 20-618 Lublin, Poland properties just after production, as well as systematic material inspection, is required for their parts. Therefore, nondestructive techniques suitable for the study of internal material structure (e.g., industrial radiography or ultrasonic testing) are being developed and improved in order to find defective products or to assess the state of fatigue. Advances in material science reveal new factors or allow understanding the already known ones that influence material properties. Many investigations show that the material surface plays an important role in their mechanical properties [1-4].

An effective method for improving the properties of the surface layer of machine elements is shot peening. During shot peening, tools (usually balls) strike the machined surface, which results in cold working of the surface layer of workpieces. Due to plastic deformation, the microhardness of the surface layer is increased and residual stresses are generated. Shot peening plays a very important role in increasing the fatigue strength of machine elements made of various materials such as iron, aluminum, titanium, magnesium, and nickel. A beneficial effect of pneumatic shot peening on the fatigue strength for specimens of titanium, aluminum, and magnesium alloys was observed [5]. Furthermore, improvement in the titanium alloy surface layer was studied [6].

It is known that shot peening changes the macroscopic material properties of many materials. The influence of shot peening conditions on Vickers microhardness and residual stresses distributions was described for spring steel [7]. The results of the research on the residual stresses in shot-peened steels and aluminum alloy specimens are presented in [8] (e.g., the maximum compressive residual stress and the depth of the residual stress field). The effectiveness of the fatigue strength improvement depends on the shot peening conditions. Therefore, the influence of impact energy and impact density on the fatigue life of the titanium alloy was investigated [9]. 
Standard methods for surface layer studying allow characterization of the results of shot peening with sufficient accuracy, but they are destructive (e.g., curvature measurements or the hole-drilling method). In order to make the studies and future industrial inspection focus on the surface layer of the materials, new nondestructive testing (NDT) methods are required. One of such promising NDT methods is positron annihilation lifetime spectroscopy (PALS) [10]. Shot peening leads to an increase in the dislocation density in the surface layer of a workpiece [5]. Its sensitivity to the size and concentration of defects predispose PALS for the study of shot-peened materials. It is confirmed by the results of our previous investigation that PALS is suitable to study the modifications of the steel surface layer [11, 12].

Special portable PALS setups are being developed for industrial application [13]. Development of a portable system implies the use of a standard positron radioactive source, which emits positrons with a broad energy spectrum. The positron energy spectrum and the properties of the studied material determine the positron implantation depth profile, which usually does not overlap the range of changes caused by shot peening. Therefore, PALS response to changes occurring in the sample is influenced by the range of the sensitivity of this method, which has to be taken into account in the interpretation of the results. In the present paper, we study the difference between PALS results obtained for various materials subjected to shot peening. Measurements for samples after various periods of shot peening allow us to examine the change in the structure of the defects during the surface treatment, as made visible by PALS.

\section{Materials}

Three standard alloys based on iron (C45 steel), aluminum (7075 alloy), and titanium (Ti6Al2Mo2Cr alloy) were chosen for the study due to their applicability in industry.
The alloy composition is presented in Table 1. Bar-shaped samples with the dimensions $4 \mathrm{~mm} \times 15 \mathrm{~mm} \times 100 \mathrm{~mm}$ were fastened to the bottom of the shot peening machine chamber. The chamber was partially filled by steel bearing balls (6 $\mathrm{mm}$ diameter) serving as a peening medium. The burnishing was performed by setting the chamber into a vertical vibratory motion (vibration amplitude $58 \mathrm{~mm}$, vibration frequency $7 \mathrm{~Hz}$ ). The time of the treatment was set to a particular value between 0.5 and $30 \mathrm{~min}$ for each set of samples. As a result, the surface layer of the samples was strain hardened to different degrees due to the impact of the bouncing balls.

\section{Experimental}

Positron annihilation lifetime spectra for the alloys under investigation were measured at room temperature by means of a conventional fast-slow delayed coincidence spectrometer. A positron source with $300-\mathrm{kBq}$ activity in the form of ${ }^{22} \mathrm{NaCl}$ sealed in 8 - $\mu$ m-thick Kapton foil was used. It was sandwiched between two identical shot-peened surfaces of alloy slim bars and mounted to a detection system. Detection of 1274-keV $\gamma$ quanta emitted from the source immediately after the $\beta^{+}$decay and giving start signals as well as the detection $511-\mathrm{keV}$ annihilation quanta serving as stop signals was realized by the use of two scintillation detector heads equipped with Photonis XP2020Q photomultipliers and $\mathrm{BaF}_{2} \phi 1.5^{\prime \prime} \times 1.0^{\prime \prime}$ crystals arranged in $180^{\circ}$ geometry. The 100-ns time range of the time-to-amplitude converter (EG\&G ORTEC 567) was chosen for the measurements. Then, the channel-width parameter was approximately equal to $0.0119 \mathrm{~ns}$. The effective time resolution (FWHM) of the PALS apparatus also including the nuclear timing electronics and the multichannel analyzer measured for ${ }^{60} \mathrm{Co}$ source did not exceed a value of about $232 \mathrm{~ns}$. The total number of events in a

Table 1 Composition of C45 steel, 7075 aluminum alloy, and Ti6Al2Mo2Cr titanium alloy

\begin{tabular}{|c|c|c|c|c|c|c|c|c|c|}
\hline Element & $\mathrm{Fe}$ & Mn & $\mathrm{C}$ & $\mathrm{Ni}$ & $\mathrm{Si}$ & $\mathrm{Cr}$ & $\mathrm{Cu}$ & $S$ & $\mathrm{P}$ \\
\hline \multicolumn{10}{|l|}{ C45 steel } \\
\hline Mass fraction $(\%)$ & 98.28 & 0.55 & 0.44 & 0.25 & 0.21 & 0.16 & 0.08 & 0.02 & 0.01 \\
\hline Element & $\mathrm{Al}$ & $\mathrm{Zn}$ & $\mathrm{Mg}$ & $\mathrm{Cu}$ & $\mathrm{Fe}$ & $\mathrm{Si}$ & $\mathrm{Mn}$ & $\mathrm{Cr}$ & $\mathrm{Ti}$ \\
\hline \multicolumn{10}{|c|}{7075 aluminum alloy } \\
\hline Mass fraction $(\%)$ & 88.62 & 5.6 & 2.5 & 1.6 & 0.4 & 0.4 & 0.3 & 0.23 & 0.2 \\
\hline Element & $\mathrm{Ti}$ & & $\mathrm{Al}$ & Mo & $\mathrm{Cr}$ & & $\mathrm{Fe}$ & $\mathrm{Si}$ & $\mathrm{C}$ \\
\hline \multicolumn{10}{|c|}{ Ti6Al2Mo2Cr titanium alloy } \\
\hline Mass fraction (\%) & 88.9 & & 6.2 & 2.2 & 2.0 & & 0.4 & 0.2 & 0.1 \\
\hline
\end{tabular}


single PALS spectrum recorded at a typical average counting rate of the order of $150 \mathrm{~s}^{-1}$ was above $10^{7}$ counts. Such statistics were sufficient to obtain reliable results on the basis of numerical analysis of the spectra.

The lifetime spectra were analyzed numerically by the LT program [14]. The model function assumed during the analysis consisted of two exponential components convoluted with a resolution curve approximated by a single Gauss function plus the constant random coincidence background. Positron annihilation in the source envelope was taken into account assuming a single exponential component with the lifetime of 374 ps. The source component intensities, $12.0 \%$ in the $\mathrm{C} 45$ steel, $10.9 \%$ in the 7075 aluminum alloy, and $11.5 \%$ in the Ti6Al2Mo2Cr titanium alloy, were calculated [15] taking into account the material composition. Additionally, the lifetime spectra were analyzed by the Bayesian method using the MELT routine [16], which allowed us to find the distribution of the intensities over the dense grid of lifetimes, without assuming the number of components.

Residual stress measurements were performed by the Davidenkov method, which consists in removal of a thin surface layer by chemical etching and measurement of the curvature change in a bar-shaped sample. The depth profile of the residual stresses was calculated using the Davidenkov-Birger equation [17]

$\sigma(z)=\frac{4 E}{3 l^{2}}\left[(h-z)^{2} \frac{\mathrm{d} g(z)}{\mathrm{d} z}-4(h-z) g(z)+2 \int_{0}^{z} g(\xi) \mathrm{d} \xi\right]$

where $E$ Young module, $l$ the length of the etched surface, $h$ the thickness of the sample before etching, $z$ the distance from the sample surface, and $g(z)$ the deflection of the sample after the removal of the layer with thickness $z$.

In order to compare the PALS and residual stress results, knowledge of the implantation depth profile was required. Because no detailed shape of the profile was required and a simple solution applicable in the industry was desired, the implantation depth profile was estimated from the equations [10]:

$n(z)=\alpha \exp (-\alpha \cdot z)$

$\alpha=\frac{1}{R}=(16 \pm 1) \frac{d}{E_{\max }^{1,43}}$,

where $z$ depth $(\mathrm{cm}), d$ average density of the material $(\mathrm{g} /$ $\left.\mathrm{cm}^{3}\right), E_{\max }$ maximum energy of positrons $(\mathrm{MeV})$, and $R$ positron implantation range.

The fact that positrons from the source are emitted in all the directions is neglected in Eq. 3. However, this causes identical distortion in all the samples, which consists in underestimation of the number of positrons annihilating near the surface. This does not affect the relative differences between the samples. The knowledge of the positron implantation depth profile allowed us to find the residual stress weighted average (over the probability of positron implantation to a particular depth) from the equation:

$\sigma_{w}=\frac{\int_{0}^{\infty}(n(z) \cdot \sigma(z))}{\int_{0}^{\infty} n(z)} \approx \frac{\sum_{i=1}\left(n_{i} \cdot \sigma_{i}\right)}{\sum_{i=1} n_{i}}$.

\section{Results and discussion}

In all the three materials under investigation and for all the shot peening times, a two-component model was sufficient to obtain a good fit of the model function to the experimental data $\left(\chi^{2} \approx 1.1\right)$. Both lifetimes are different in each sample, but shot peening does not change them significantly (Fig. 1). The exceptions visible for the short shot peening times have to be considered taking into account that the discrepancies are usually connected with the very low value of the respective intensity. It makes them highly sensitive to systematic errors (e.g., an imprecise determination of source correction). Therefore, any interpretation of these changes is questionable. However, the origin of the particular components in each sample can be deduced, mostly unambiguously, from the average lifetimes (Table 2).

In spite of the large iron contents in the $\mathrm{C} 45$ steel, a component with a lifetime close to $100 \mathrm{ps}$, typical for bulk iron $[18,19]$, is not present in the spectra. The shortest observed lifetime $\tau_{1}=151 \mathrm{ps}$ indicates that all positrons are trapped in defects. This dominating component $\left(I_{1}>95 \%\right)$ can originate from positrons trapped in monovacancies associated with edge dislocation lines in iron [20] or vacancysolute complexes for steel components (e.g. V-Cr or V-Cu) [21]. Such a result is typical for steels [22-24]. The lifetime $\tau_{2}=407 \mathrm{ps}$ of the long-lived component is longer than that usually observed in deformed steel and iron [19, 24]. Its origin is most probably related to large vacancy clusters consisting of more than 15 vacancies [25].

The lifetime $\tau_{1}=210 \mathrm{ps}$ of the short-lived component in the $7075 \mathrm{Al}$ alloy is representative for aluminum alloys [2629]. Its value lies clearly below $\sim 250$ ps expected for $\mathrm{Al}$ monovacancy $[18,25]$. A standard explanation for this discrepancy is the contribution of positron annihilation in vacancies bound to different atoms other than the $\mathrm{Al}$ ones present in the alloy [27-29]. The resulting lifetimes would be too similar to isolate particular components. However, considering the single components with the greatest fraction in the investigated alloy, only positron lifetime in $\mathrm{Cu}$ monovacancy is significantly shorter than the lifetime in $\mathrm{Al}$ monovacancy. Assuming after others that lifetime in vacancy-solute complex can be estimated based on that 


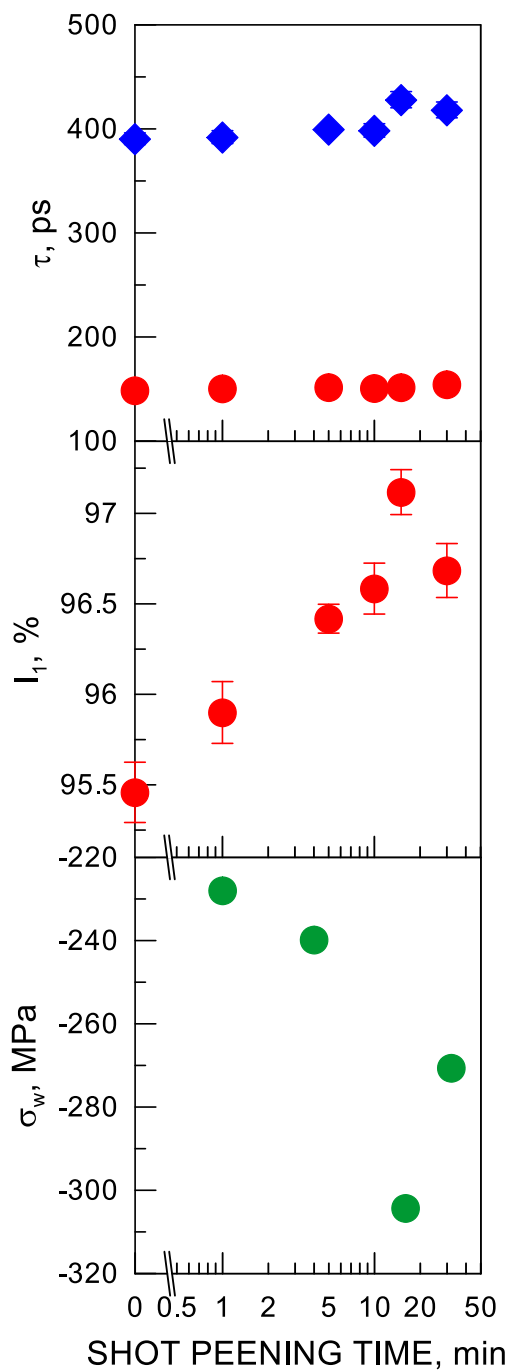

Fig. 1 Parameters of two components found in the positron annihilation lifetime spectra of the $\mathrm{C} 45$ steel (left), the 7075 aluminum alloy (middle), and the Ti6Al2Mo2Cr titanium alloy (right): lifetimes $\left(\tau_{1}\right.$, $\left.\tau_{2}\right)$, intensity $\left(I_{1}\right)$ of the short-lived component obtained for samples

Table 2 Lifetimes averaged over all shot peening times and their standard deviation for the studied samples

\begin{tabular}{lllll}
\hline Sample & $\tau_{1}(\mathrm{ps})$ & $s_{\tau 1}(\mathrm{ps})$ & $\tau_{2}(\mathrm{ps})$ & $s_{\tau 2}(\mathrm{ps})$ \\
\hline C45 steel & 151 & 2 & 407 & 14 \\
7075 aluminum alloy & 210 & 8 & 350 & 13 \\
Ti6Al2Mo2Cr titanium alloy & 189 & 5 & 460 & 44 \\
\hline
\end{tabular}

related to the bulk material, only the lifetime in $\mathrm{V}-\mathrm{Cu}$ is short enough to give a contribution, which shortens the lifetime to the value observed in the experiments. Therefore, the observed lifetime would indicate a high probability of positron trapping in the neighborhood of $\mathrm{Cu}$ atoms. On the other hand, there is a simpler interpretation of the results. Shorter lifetimes $\sim 225$ and $\sim 190$ ps are

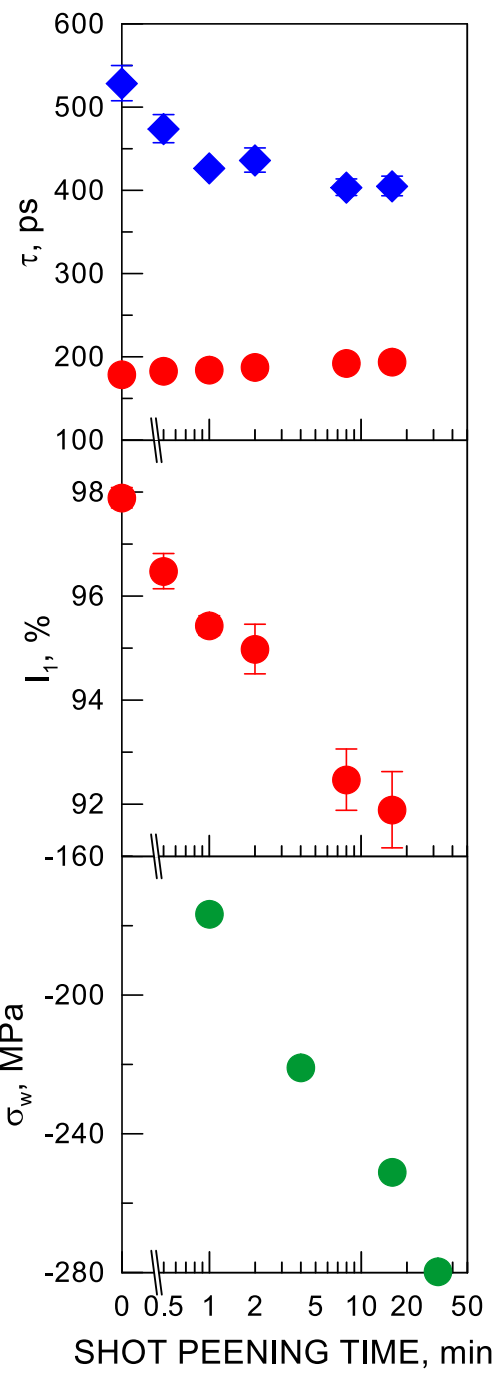

after various times of shot peening. The intensity of the long-lived component can be calculated by subtracting the $I_{1}$ value from $100 \%$. The PALS results were compared to the corresponding residual stress weighted average $\left(\sigma_{w}\right)$

expected for a single vacancy or a jog on the dislocation line and for dislocation loops, respectively [30]. Their contributions can be more essential than the ones from the vacancy-solute complexes. The second component lifetime $\tau_{2}=350 \mathrm{ps}$ is characteristic for much smaller vacancy clusters ( $\sim 6$ vacancies) than the ones found in the steel [25].

There is a shortage of both theoretical and experimental data about the lifetimes in $\mathrm{Ti}$ alloys with high titanium contents. Similar to the discussed steel and the $\mathrm{Al}$ alloy, the shortest lifetime observed in Ti6Al2Mo2Cr samples is longer than the Ti bulk lifetime (140-150 ps) and shorter than the lifetime of positrons trapped in monovacancies (220-240 ps) calculated or observed in high-purity Ti or its alloys with contents close to $50 \%[18,31-33]$. As in previous cases, the observed lifetime can be attributed to 
vacancies on dislocation lines or in the neighborhood of alloy components other than the Ti atoms. There also exists a possibility that the layer of titanium oxides on the sample surface gives contribution with the lifetime of 190-200 ps [34]. It is probably impossible to distinguish between all these contributions with similar lifetimes, and in consequence, the mean lifetime of $189 \mathrm{ps}$ is observed. The second component indicates that vacancy clusters are also present in the Ti alloy.

The analysis of the origin of the components in the investigated samples, which was presented above, allows us to explain the character of changes caused by shot peening from positron lifetime measurement (Fig. 1).

The most pronounced and systematic change is visible in the relative intensities of both lifetime components. In general, the intensity of the first component $\left(I_{1}\right)$ increases with the shot peening time in the steel and the $\mathrm{Al}$ alloy. An exception from the monotonic rise is a discontinuity visible for $I_{1}$ in spectra after 5 and 10 min of shot peening in the case of the $\mathrm{Al}$ alloy and, to a lesser degree, in spectra after 15 and $30 \mathrm{~min}$ of shot peening in the case of the steel. Nevertheless, it is hard to determine whether these results reflect a sample structure change or random dispersion of the results. This second possibility is supported by the lifetime values, i.e., the difference between $\tau_{2}$ and $\tau_{1}$ is larger if $I_{1}$ values lie above the observed trend lines and vice versa. An opposite tendency in the $I_{1}$ changes (i.e., a decrease with the rise in the shot peening time) is observed in the case of the Ti alloy.
In order to find the cause of the difference between the shot peening influence on positron lifetime spectra in the steel or the Al alloy and Ti alloy, the PALS results are compared to the average of residual stress $\left(\sigma_{w}\right)$ weighted over the positron implantation profile (Fig. 1). The weighted average of residual stresses generally decreases (i.e., compressive residual stresses, which are denoted as negative values in the Davidenkov method, increase) with the increasing shot peening time, and there is no qualitative difference between the studied samples. A change in the decreasing tendency can be found in the steel, which was processed for more than $20 \mathrm{~min}$. This result is confirmed by the previous findings obtained for different samples made of the same material [11]. It also confirmed that the decrease in $I_{1}$ between 15 and 30 min was not caused by a statistical dispersion of the results. However, it was not found during the previous studies of the same kind. There is no confirmation of $I_{1}$ discontinuity in the $\mathrm{Al}$ alloy. The residual stress studies suggest that further changes in the surface layer of the material penetrated by positrons are insignificant for a shot peening time longer than $5 \mathrm{~min}$. It is worth noticing that the $\sigma_{w}$ values are about ten times smaller in the $\mathrm{Al}$ alloy than in the other studied materials. The simplest relation between $\sigma_{w}$ and the shot peening time is found for the Ti alloy giving no support for the reversed $I_{1}$ tendency. This proves that taking into account the weighted average value of the residual stress only is insufficient. Therefore, a more detailed study of the residual stress depth distribution is required.

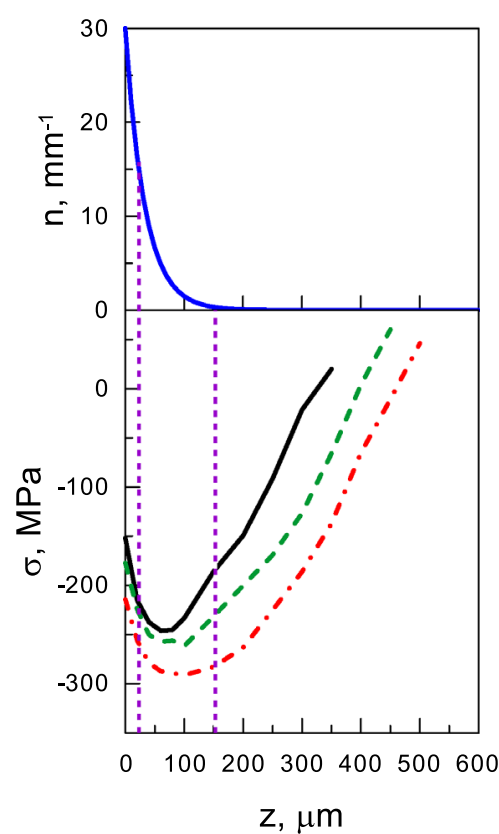

Fig. 2 Depth distribution of residual stresses $(\sigma)$ in the C45 steel (left), the 7075 aluminum alloy (middle), and the Ti6Al2Mo2Cr (right) after about 1 (solid line), 5 (dashed line), and 30 (dot-dash line) minutes of shot peening. The distribution is compared to the
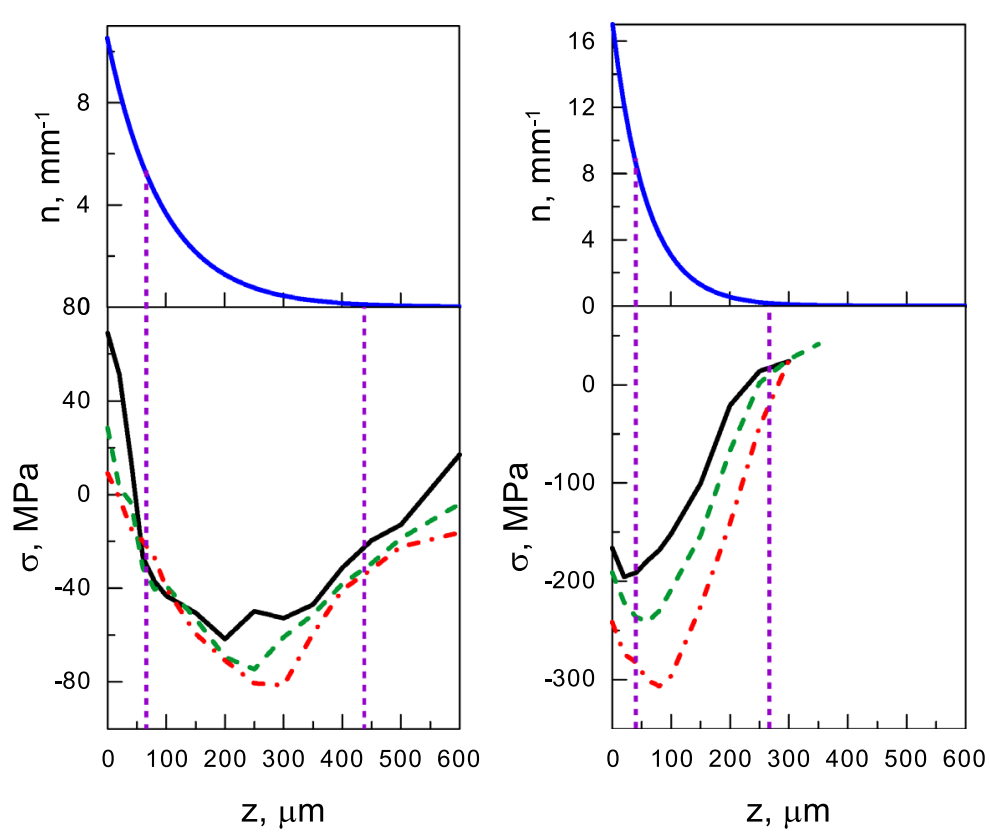

positron implantation depth profile $(n)$ in the particular materials. Two vertical dotted lines at the smaller and larger depths represent approximate ranges below which 50 and $99 \%$ of positrons annihilate, respectively 
The comparison between residual stress depth distributions and positron implantation profiles estimated from Eq. 2 is presented in Fig. 2 for all the studied samples. The residual stress depth distributions are u-shaped for all of the samples. They are very similar for the steel and the Ti alloy. Both distributions start with a large negative value on the surface indicating existence of compressive residual stresses, a decrease within $100 \mu \mathrm{m}$ below the surface, and then an increase to about zero in the range of another several hundred micrometers. For both the steel and $\mathrm{Ti}$ alloy samples, an increase in the shot peening time results in a shift of the distributions toward negative values almost without changing their shape. The distribution in the steel is only a little widened, while the one in the Ti alloy becomes slightly narrower. The residual stress depth distribution in the Al alloy is quite different. It starts from positive values on the surface, which indicate tensile stresses. The layer where residual stress decreases is much deeper, whereas the lowest value lies 4-5 times above analogical values in the steel or the Ti alloy. Furthermore, the changes caused by the increase in the shot peening time are much smaller compared with the other samples, although the changed direction seems to agree with the ones observed in other materials.

The positron implantation range is the smallest in the steel $[R=33(2) \mu \mathrm{m}]$, about two times greater in the $\mathrm{Ti}$ alloy $[R=58(4) \mu \mathrm{m}]$, and three times greater in the $\mathrm{Al}$ alloy $[R=95(8) \mu \mathrm{m}]$. On the other hand, the minimum of residual stress distribution (i.e., the greatest compressive residual stress) is roughly at the same depth in both the steel and the Ti alloy (50-100 $\mu \mathrm{m})$ but at about three times greater depth $(200-300 \mu \mathrm{m})$ in the Al alloy. Thus, positrons penetrate mostly the surface layer where residual stress decreases with depth in the steel and the Al alloy, while in the Ti alloy, they annihilate almost in the whole layer where residual stress is present. This difference between the $\mathrm{Ti}$ alloy and the other materials allows us to explain the opposite inclination of $I_{1}$ changes with the increasing shot peening time for this material.

The appearance and the increase in residual stresses caused by shot peening testify that the distribution of the defects in the samples becomes depth dependent. The concentration of defects of a particular kind and size, which are present in the material, changes with the distance from the sample surface [22]. Due to the exponential shape of the positron implantation profile, there is a different contribution to the intensity of the positron component from the defects near the sample surface in comparison with the same defects present in the depth of the sample. Therefore, the change in intensity can be a result of either the change in the total concentration of the defects attributed to this component or the change in the concentration profile in the direction perpendicular to the sample surface. Taking into account this findings for the present results, we can conclude that the changes in $I_{1}$ observed in the studied samples do not necessarily prove that the total concentration ratio of the smaller free volumes (monovacancies) to the larger ones (vacancy clusters) increases in the steel and the Al alloy, but decreases in the $\mathrm{Ti}$ alloy. Another possibility is that due to the shot peening, large defects are moved away from the surface, while most of the small ones remain near the surface. Thus, the dissimilarity of the PALS results for the Ti alloy may be a result of a greater scope of data collected in this material rather than a different consequence of shot peening. Even though the residual stress measurement does not allow distinguishing between the sizes of the defects, the difference between the scope of the positron study in the Ti alloy and the other alloys can be detected by this method. The contribution of the compressive residual stress present virtually above the positron range (available only to $1 \%$ of positrons) to the whole residual stress was estimated from its depth

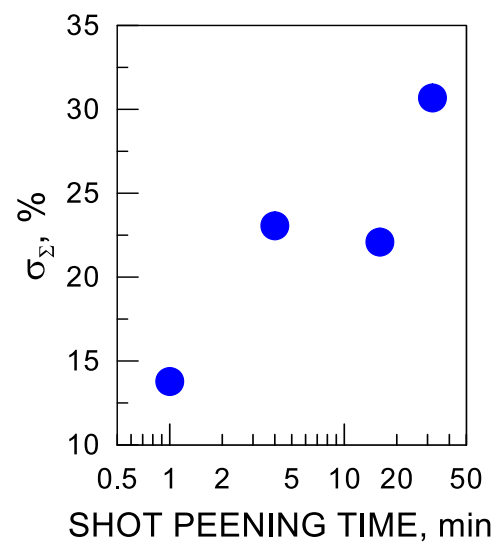

Fig. 3 Relative contribution of the compressive residual stress $\left(\sigma_{\Sigma}\right)$ above the range below which $99 \%$ of positrons annihilate (marked in Fig. 2) for the C45 steel (left), the 7075 aluminum alloy (middle), and
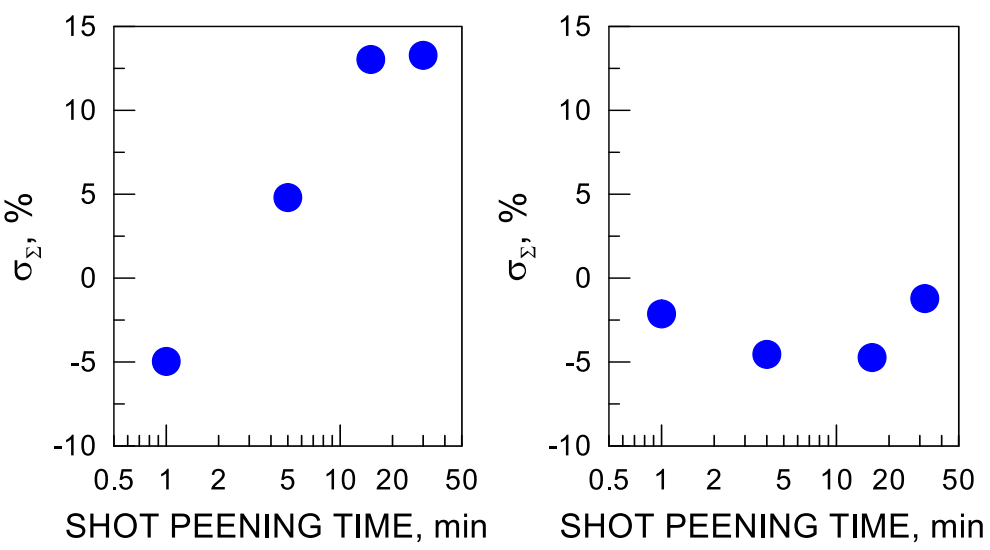

the Ti6A12Mo2Cr (right) samples after various times of shot peening. The negative values mean predominance of the tensile residual stress 
distribution (Fig. 3). With the time of shot peening, the contribution clearly increases in the steel and Al alloy, while no such effect is observed in the Ti alloy. The interpretation presented previously is supported by the fact that there is no other evolution of the residual stresses in the Ti alloy compared with the other material, which could be expected if the distribution of the defects is substantially different. Thus, if shot peening influences the defect distribution in all the samples in a similar way, we can describe the mechanism of the defect formation in greater detail taking advantage of the differences in the positron range. Hence, we can determine not only that shot peening causes formation of a higher number of large defects in comparison with small ones, as observed in the Ti alloy, but also that the larger defects are formed far from the surface, beyond the positron ranges in the steel and the $\mathrm{Al}$ alloy samples.

Other information about the size distribution of defects can be obtained from the analyses of the PALS spectra performed by MELT. Such analyses were performed in order to determine whether the dispersion of the results observed in the $\mathrm{Al}$ alloy was dependent on the analysis method. This aim could not be achieved because the lifetime histograms obtained from MELT showed very wide lifetime distributions (Fig. 4). Such distributions do not allow separation of the two components, which does not let us determine their intensities. However, an interesting dependence between a lifetime distribution and a shot peening time is visible in the histograms. The positron lifetimes are widely distributed between 170 and 300 ps forming virtually one asymmetric peak before shot peening. However, the processing causes formation of two wellseparated peaks instead of an initial one. The cause of such a change is probably systematic disappearance of the defects, in which the positron lifetime is about $230 \mathrm{ps}$, in consequence of the shot peening. The lifetime value allows us to identify these defects as monovacancies $(250 \mathrm{ps})$ or monovacancies on the dislocation line ( $225 \mathrm{ps})$. Keeping in mind the previous findings, we have to notice that this effect is observed only close to the surface, and the changes may be different in the deeper layers of the sample. No similar changes were found in the other samples.
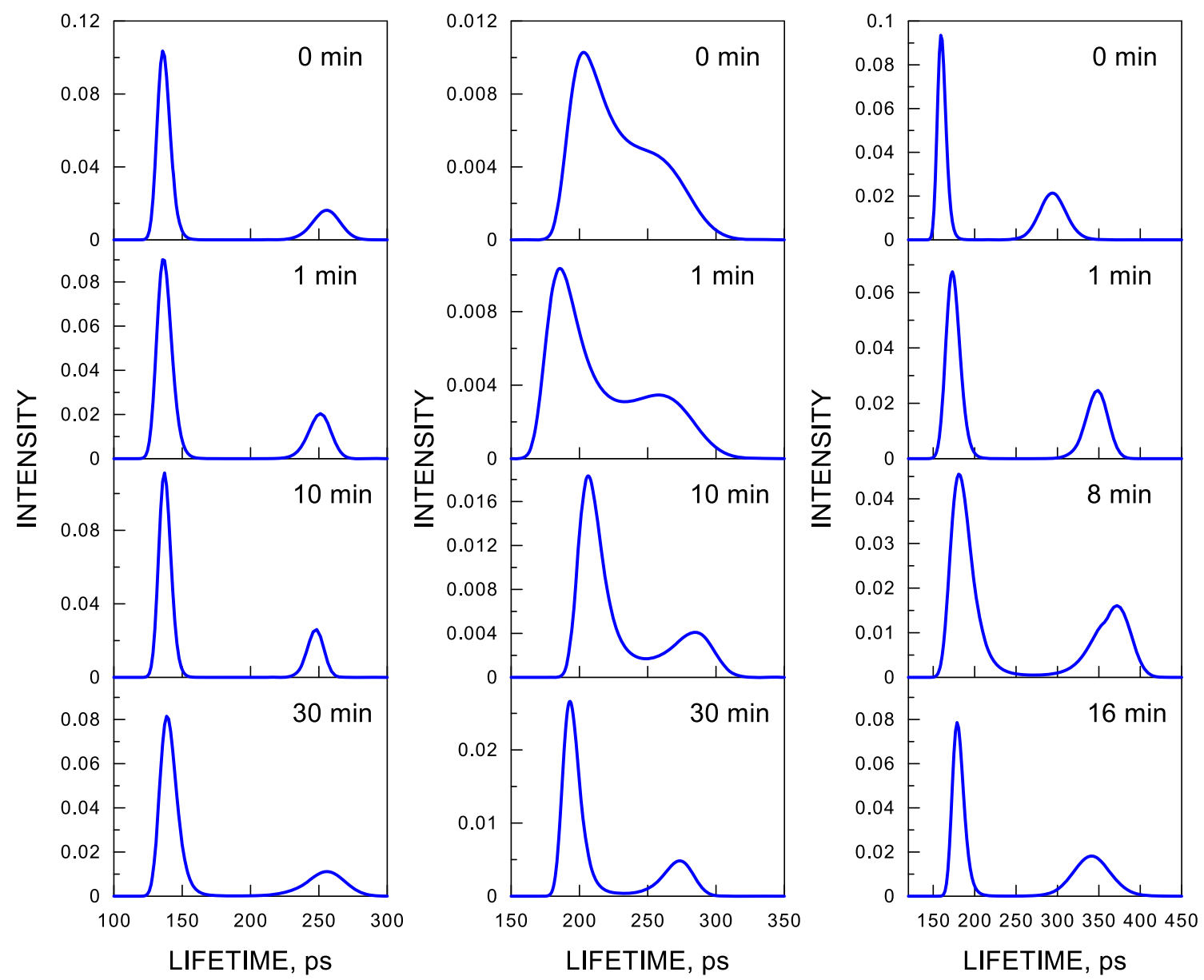

Fig. 4 Histograms of intensities on the dense lifetime grid obtained by MELT for the C45 steel (left), the 7075 aluminum alloy (middle), and Ti6Al2Mo2Cr (right) for an unburnished sample and after the time of shot peening, which is specified in the figure 
Table 3 Lifetimes and the intensities of the shot-peened for $10 \mathrm{~min}$ samples ( $8 \mathrm{~min}$ in the case of the titanium alloy) obtained using two different methods for spectra analysis provided by LT and MELT

\begin{tabular}{lllllrr}
\hline Sample & LT & & \multicolumn{1}{l}{ MELT } & \\
\cline { 2 - 3 } & $\tau_{1}(\mathrm{ps})\left[I_{1}(\%)\right]$ & $\tau_{2}(\mathrm{ps})\left[I_{2}(\%)\right]$ & & $\tau_{1}(\mathrm{ps})\left[I_{1}(\%)\right]$ & $\tau_{2}(\mathrm{ps})\left[I_{2}(\%)\right]$ & $\tau_{3}(\mathrm{ps})\left[I_{3}(\%)\right]$ \\
\hline C45 steel & $151[96.6]$ & $399[3.4]$ & & $145[84.8]$ & $247[15]$ & $940[0.2]$ \\
7075 aluminum alloy & $217[91.7]$ & $336[8.3]$ & & $211[78.1]$ & $279[21.8]$ & $977[0.1]$ \\
Ti6Al2Mo2Cr titanium alloy & $192[92.5]$ & $403[7.5]$ & & $186[77.2]$ & $360[22.6]$ & $1450[0.2]$ \\
\hline
\end{tabular}

The MELT results presented in Fig. 4 show a systematic shift toward the short lifetimes in comparison with the LT results. The cause of such discrepancy is the long-lived and low-intensity component found by MELT, the example of which is shown in Table 3 for samples that were shotpeened for about $10 \mathrm{~min}$. The origin of the component of such low intensity can be either positron annihilation in microcracks, which has already been observed in steels [12], or the undesired distortion of the spectra, e.g., the detection of scattered annihilation radiation. An attempt to take this component into account during LT analysis results in better agreement with the MELT results, but also the uncertainties of the parameters become 2-3 times larger, while $\chi^{2}$ decreases only by 2-3\%. Therefore, only the twocomponent LT analysis allows drawing conclusions about the changes caused by shot peening.

\section{Conclusions}

Analyzing the PALS spectra for the studied samples in terms of two discrete (i.e., exponential) positron lifetime components is definitely an oversimplification. The MELT results show that at least three discrete components are required to find what kinds of defects are responsible for the changes observed in the spectra of the studied alloys. Unfortunately, such a high number of components with close lifetimes cannot be distinguished using the standard methods of spectra analysis. Even for high-statistic spectra, keeping a reasonable uncertainty in the measured values is impossible. Nevertheless, a two-component approximation of the small and large defects allows us to find an interesting relationship between the shot peening time and the component intensities.

A discrepancy between the steel and the $\mathrm{Al}$ alloy, where the intensity of the short-lived, dominating component increases with the shot peening time and the Ti alloy shows an opposite relation, is clearly visible. From the fact that the discrepancy is not reflected by the macroscopic residual stresses, we conclude that this effect is related to PALS properties. The positron range in relation to the depth of the structure changes caused by shot peening seems to be the best explanation for the observed phenomenon. Additional conclusions about the defect distribution can be found taking into consideration the positron range. Hence, we deduce that shot peening causes migration of large defects (i.e., vacancy clusters) away from the surface, while only smaller ones (i.e., monovacancies or vacancies on dislocation lines) remain close to the surface. Because all positrons are trapped in defects, it is possible to observe relative changes only.

It would be interesting to verify the findings presented above by the use of a variable energy positron beam, but currently there is no setup that would allow us to obtain monoenergetic positrons with high-enough energy to perform this experiment. It is also possible to perform analogical measurements removing subsequent layers from the sample surface, but there is no guarantee that this process will not influence the sample structure. An interesting alternative for the positron source is ${ }^{44} \mathrm{Ti}$, which emits positrons with an about three times higher energy than ${ }^{22} \mathrm{Na}$. If our considerations concerning the positron range are correct, a measurement performed with a ${ }^{44} \mathrm{Ti}$ source should show a decrease in the short-lived intensity component also in steel and the $\mathrm{Al}$ alloy.

Acknowledgments Financial support of Structural Funds in the Operational Programme-Innovative Economy (IE OP) financed from the European Regional Development Fund-Project "Modern material technologies in aerospace industry", Nr POIG.01.01.02-00015/08-00 is gratefully acknowledged.

Open Access This article is distributed under the terms of the Creative Commons Attribution 4.0 International License (http:// creativecommons.org/licenses/by/4.0/), which permits unrestricted use, distribution, and reproduction in any medium, provided you give appropriate credit to the original author(s) and the source, provide a link to the Creative Commons license, and indicate if changes were made.

\section{References}

1. D.T. Asquith, A.L. Yerokhin, J.R. Yates, A. Matthews, Thin Solid Films 516, 417 (2007)

2. A.L.M. Carvalho, H.J.C. Voorwald, Mater. Sci. Eng., A 505, 31 (2009) 
3. V. Azar, B. Hashemi, M. Rezaee Yazdi, Surf. Coat. Technol. 204, 3546 (2010)

4. K. Oguri, J. Mater. Process. Tech. 211, 1395 (2011)

5. L. Wagner, Mater. Sci. Eng., A 263, 210 (1999)

6. H. Boeckels, L. Wagner, in Shot Peening and Other Mechanical Surface Treatment, ed. by V. Schultze, A. Niku-Lari. Proceedings of the 9th International Conference on Shot Peening (Paris, 2005), p. 332-337

7. A. Tange, K. Ando, Mater. Sci. Technol. 18, 642 (2002)

8. S. Wang, Y. Li, M. Yao, R. Wang, J. Mater. Process. Tech. 73, 64 (1998)

9. K. Zaleski, Eksploatacja i Niezawodnosc. Maint. Reliab. 4, 65 (2009)

10. P. Hautojärvi, Positrons in Solids, 1st edn. (Springer, Berlin, 1979)

11. R. Zaleski, K. Zaleski, Acta Phys. Polon. A 110, 739 (2006)

12. R. Zaleski, M. Gorgol, K. Zaleski, Physics Procedia 35, 92 (2012)

13. M. Yamawaki, Y. Kobayashi, K. Hattori, Y. Watanabe, Jpn. J. Appl. Phys. 50, 086301 (2011)

14. J. Kansy, Nucl. Instrum. Methods Phys. Res., Sect. A 374, 235 (1996)

15. T. Goworek, W. Górniak, J. Wawrysczuk, Nucl. Instrum. Methods Phys. Res., Sect. A 321, 560 (1992)

16. A. Shukla, M. Peter, L. Hoffmann, Nucl. Instrum. Methods Phys. Res., Sect. A 335, 310 (1993)

17. I.A. Birger, Residual Stresses (Mashgiz, Moscow, 1963)

18. J.M.C. Robles et al., J. Phys.: Condens. Matter 19, 176222 (2007)

19. H.F.M. Mohamed, J. Kwon, Y.-M. Kim, W. Kim, Nucl. Instrum. Methods Phys. Res., Sect. B 258, 429 (2007)

20. E. Kuramoto, T. Tsutsumi, K. Ueno, M. Ohmura, Y. Kamimura, Comp. Mater. Sci. 14, 28 (1999)
21. J. Kuriplach, O. Melikhova, C. Domain, C.S. Becquart, D. Kulikov, L. Malerba, M. Hou, A. Almazouzi, C.A. Duque, A.L. Morales, Appl. Surf. Sci. 252, 3303 (2006)

22. X.W. Wang, J.Y. Wang, L.Y. Xiong, G. Liu, Mater. Sci. Forum 445-446, 210 (2004)

23. G. Aldi, P. Coppa, A. Dupasquier, Lett. Nuovo Cimento 39, 151 (1984)

24. W. Rudzińska, J. Pająk, M. Szuszkiewicz, G. Bujnarowski, A.A. Kluza, Acta Phys. Polon. A 110, 691 (2006)

25. M.J. Puska, R.M. Nieminen, J. Phys. F: Met. Phys. 13, 333 (1983)

26. E. Dryzek, Acta Phys. Polon. A 107, 753 (2005)

27. O. Melikhova, J. Čížek, I. Procházka, J. Kuriplach, I. Stulíková, J. Faltus, Mater. Struct. 8, 61 (2001)

28. T.E.M. Staab, E. Zschech, R. Krause-Rehberg, J. Mater. Sci. 35, 4667 (2000)

29. A. Somoza, A. Dupasquier, I.J. Polmear, P. Folegati, R. Ferragut, Phys. Rev. B: Condens. Matter Mater. Phys. 61, 14454 (2000)

30. H. Häkkinen, S. Mäkinen, M. Manninen, Phys. Rev. B: Condens. Matter Mater. Phys. 41, 12441 (1990)

31. W. Deng, D. Xiong, J. Wang, L. Xiong, M. Cao, C. Lung, J. Mater. Sci. Technol. 19, 164 (2003)

32. M. Misheva, N. Djourelov, T. Kotlarova, D. Elenkov, G. Passage, Thin Solid Films 283, 26 (1996)

33. Y. Nagai, T. Toyama, Z. Tang, M. Hasegawa, H. Matsumoto, S. Hanada, Scr. Mater. 54, 1751 (2006)

34. A.A. Valeeva, A.A. Rempel, W. Sprengel, H.E. Schaefer, Phys. Solid State 51, 924 (2009) 